\title{
Effect of hindlimb unweighting on tissue blood flow in the rat
}

\author{
K. S. McDONALD, M. D. DELP, AND R. H. FITTS \\ Biology Department/Biological and Biomedical Research Institute, Marquette University, Milwaukee, \\ Wisconsin 53233; and Exercise Biochemistry Laboratory, University of Georgia, Athens, Georgia 30602
}

\begin{abstract}
McDonald, K. S., M. D. DelP, AND R. H. FitTs. Effect of hindlimb unweighting on tissue blood flow in the rat. J. Appl. Physiol. 72(6): 2210-2218, 1992.-The purpose of this study was to characterize the distribution of blood flow in the rat during hindlimb unweighting ( $\mathrm{HU}$ ) and post-HU standing and exercise and examine whether the previously reported (Witzmann et al., J. Appl. Physiol. 54: 1242-1248, 1983) elevation in anaerobic metabolism observed with contractile activity in the atrophied soleus muscle was caused by a reduced hindlimb blood flow. After either 15 days of HU or cage control, blood flow was measured with radioactive microspheres during unweighting, normal standing, and running on a treadmill (15 $\mathrm{m} / \mathrm{min}$ ). In another group of control and experimental animals, blood flow was measured during preexercise (PE) treadmill standing and treadmill running $(15 \mathrm{~m} / \mathrm{min})$. Soleus muscle blood flow was not different between groups during unweighting, PE standing, and running at $15 \mathrm{~m} / \mathrm{min}$. Chronic unweighting resulted in the tendency for greater blood flow to muscles composed of predominantly fast-twitch glycolytic fibers. With exercise, blood flow to visceral organs was reduced compared with $\mathrm{PE}$ values in the control rats, whereas flow to visceral organs in 15-day HU animals was unaltered by exercise. These higher flows to the viscera and to muscles composed of predominantly fast-twitch glycolytic fibers suggest an apparent reduction in the ability of the sympathetic nervous system to distribute cardiac output after chronic HU. In conclusion, because 15 days of HU did not affect blood flow to the soleus during exercise, the increased dependence of the atrophied soleus on anerobic energy production during contractile activity cannot be explained by a reduced muscle blood flow.
\end{abstract}

energy metabolism; muscle atrophy

SPACEFLIGHT is known to produce bone demineralization, atrophy to antigravity muscles, and cardiovascular changes $(4,36)$. Land-based animal models, including hindlimb immobilization (HI) and hindlimb unweighting (HU), have been developed to simulate weightlessness conditions. These models have proven to elicit changes in bone composition and muscle structure, function, and biochemistry that closely mimic those observed with microgravity $(13,14,16,23,26,28)$. In addition, HU elicits hemodynamic changes-including a transient increase in central venous pressure (31), natriuresis and diuresis (6), reduction in blood volume $(11,30)$, and a decrease in exercise capacity $(10,30)$-similar to those induced by spaceflight (4).

The effects of simulated weightlessness on muscle properties have been well characterized. Whole muscle and single fiber studies have shown the postural, anti- gravity soleus muscle to be most affected $(13,14)$. Both HU and HI caused a significant reduction in soleus peak twitch and tetanic tension $(13,34,37)$, and an increased fatigability $(27,37)$. After $\mathrm{HI}$, the atrophied soleus displayed greater glycogen and ATP usage and lactate production during contractile activity (37). In addition, soleus muscle fiber glycogen, ATP, and creatine phosphate stores are increased after $2 \mathrm{wk} \mathrm{HU}(17,18)$. These results suggest a shift in the atrophied soleus toward a greater dependence on anaerobic metabolism. This shift occurred despite an increased oxidative capacity (per fiber weight) in the soleus after HU and HI (12). Thus the purpose of this study was to use the radiolabeled microsphere technique to 1) characterize the distribution of blood flow during $\mathrm{HU}$ and post-HU standing and exercise and 2) examine whether the increased reliance on anaerobic energy production of the atrophied soleus during contractile activity results from a decreased muscle blood flow.

The results demonstrate that, compared with the control standing value, blood flow to the soleus was reduced during unweighting in both the control (acute 10-min unweighting) and the 15-day $\mathrm{HU}$ group and the 15-day $\mathrm{HU}$ group with normal standing. No differences between groups were observed in soleus muscle blood flow during a 5-min bout of treadmill exercise; however, the redistribution of visceral flow with the onset of exercise was attenuated after 15 days of $\mathrm{HU}$.

\section{METHODS}

Animals. Male Sprague-Dawley rats (250-275 g) were obtained and taught to run on a motor-driven treadmill (10 $\mathrm{min} /$ day, $20 \mathrm{~m} / \mathrm{min}$ for 10 days) and then randomly assigned to the $\mathrm{HU}$ or cage control group. The hindlimbs of the $\mathrm{HU}$ animals were partially elevated for 15 days with a harness attached to the proximal two-thirds of the tail, as previously described in detail (13). The height of hindlimb elevation was adjusted to prevent the hindlimbs from touching supportive surfaces. The forelimbs maintained contact with a grid floor, which allowed the animal full range of motion. The $\mathrm{HU}$ animals were fed Purina rat chow and water ad libitum, whereas control rats were pair fed to maintain weights similar to those of the $\mathrm{HU}$ group. Both groups were housed at $23^{\circ} \mathrm{C}$ with a 12:12-h light-dark cycle per day.

Surgical procedures. After 15 days the HU and control rats were anesthetized with methoxyflurane (Metofane) anesthesia. The HU group were anesthetized while re- 
. 
maining unweighted to avoid any weight-bearing muscular activity. A catheter (Silastic; $0.6 \mathrm{~mm}$ ID, $1.0 \mathrm{~mm} \mathrm{OD)}$ was surgically implanted in the ascending aorta via the right carotid artery as previously described (20). This catheter was subsequently used for recording arterial pressure and the infusion of radiolabeled microspheres for blood flow measurements. A second catheter (polyurethane; $0.36 \mathrm{~mm}$ ID, $0.84 \mathrm{~mm}$ OD) was inserted $\sim 4 \mathrm{~cm}$ into the caudal artery as described by Chiueh and Kopin (4) with several modifications. This catheter was used to withdraw blood for the blood flow measurements. Both catheters were filled with a heparinized $(200 \mathrm{U} / \mathrm{ml})$ saline solution and externalized on the dorsal cervical region.

Experimental protocol. After $12 \mathrm{~h}$ of recovery from catheter implantations, control and $\mathrm{HU}$ animals were instrumented for blood flow measurements. The hindlimbs of the control rats were elevated, and after a 10 -min equilibration period, blood flow was measured in both groups. The rats were then lowered to a standing hindlimbweight-bearing position, and blood flow was determined after $10 \mathrm{~min}$ of normal standing. Finally, the animals were run at $15 \mathrm{~m} / \mathrm{min}$ on a motorized treadmill, and blood flow measured at the 5 th min of running. In another group of control and experimental animals, the rats were placed directly on the treadmill after the 12 -h recovery from catheter implantations, and blood flow measured after $10 \mathrm{~min}$ of standing. In the text, this measurement is referred to as the preexercise (PE) blood flow. In this group, a second blood flow measurement was made during the 5 th $\mathrm{min}$ of running at $15 \mathrm{~m} / \mathrm{min}$. Because the blood flow measurements during exercise were not affected by the type of PE standing (normal vs. treadmill), these values were pooled (see Table 3). After the final blood flow measurement, pentobarbital sodium was infused through the carotid catheter to induce deep anesthesia. The rats were then decapitated; and tissue samples were excised, weighed, and placed in counting vials for flow determination.

Blood flow measurements. Radiolabeled $\left({ }^{85} \mathrm{Sr},{ }^{113} \mathrm{Sn}\right.$, and ${ }^{153} \mathrm{Gd}$ ) microspheres (New England Nuclear) with a $15-\mu \mathrm{m}$ diam were used for blood flow measurements as previously described (21). Radioactivity of the samples was measured with a gamma counter (Packard AutoGamma 5780), and flows were computed (IBM-PC computer) from counts per minute and tissue wet weights. To ensure that microsphere-blood mixing was adequate, flows to the right and left kidneys were compared for each animal. Mixing was considered adequate if both kidney flows were within $15 \%$ of each other.

Central hemodynamic measurements. Mean arterial pressure and heart rate were recorded from the carotid catheter just before each microsphere infusion using a pressure transducer (Narco Bio-Systems) and recorder (Narco physiograph). Only mean arterial pressure was reported because of the damping effect of the catheters. Heart rates were determined from the pulsatile pressure recordings.

Data analysis. A one-way analysis of variance with repeated measures was used to compare heart rates, mean arterial pressures, and blood flows within tissues across unweighting, standing, $\mathrm{PE}$, and $15 \mathrm{-m} / \mathrm{min}$ running conditions. Duncan's new multiple-range test was then used
TABLE 1. Tissue weights

\begin{tabular}{lcccccc}
\hline & \multicolumn{2}{c}{$\begin{array}{c}\text { Tissue Weight, } \\
\text { mg }\end{array}$} & & \multicolumn{2}{c}{$\begin{array}{c}\text { Tissue Weight/Body } \\
\text { Weight, mg/g }\end{array}$} \\
\cline { 2 - 3 } \cline { 6 - 7 } \multicolumn{1}{c}{ Tissue } & $\mathrm{C}$ & $\mathrm{HU}$ & & $\mathrm{C}$ & $\mathrm{HU}$ \\
\hline Soleus & $152 \pm 4$ & $88 \pm 3^{*}$ & & $0.48 \pm 0.01$ & $0.30 \pm 0.01^{*}$ \\
Plantaris & $352 \pm 8$ & $298 \pm 8^{*}$ & & $1.12 \pm 0.02$ & $1.00 \pm 0.02^{*}$ \\
Gastrocnemius & $1,775 \pm 44$ & $1,490 \pm 38^{*}$ & & $5.64 \pm 0.13$ & $4.99 \pm 0.10^{*}$ \\
Tibialis anterior & $676 \pm 16$ & $659 \pm 11$ & & $2.14 \pm 0.04$ & $2.21 \pm 0.02$ \\
EDL & $174 \pm 4$ & $169 \pm 3$ & & $0.55 \pm 0.01$ & $0.57 \pm 0.01$ \\
Vastus intermedius & $120 \pm 7$ & $86 \pm 4^{*}$ & & $0.38 \pm 0.02$ & $0.29 \pm 0.01^{*}$ \\
Vastus lateralis & $1,202 \pm 28$ & $1,107 \pm 27^{*}$ & & $3.82 \pm 0.09$ & $3.74 \pm 0.07$ \\
Adrenal medulla & $36 \pm 2$ & $40 \pm 2$ & & $0.11 \pm 0.01$ & $0.14 \pm 0.01^{*}$ \\
Adrenal cortex & $25 \pm 2$ & $29 \pm 2$ & & $0.07 \pm 0.01$ & $0.10 \pm 0.01^{*}$ \\
Left kidney & $1,285 \pm 28$ & $1,317 \pm 24$ & & $4.10 \pm 0.12$ & $4.44 \pm 0.11$ \\
Spleen & $650 \pm 21$ & $688 \pm 33$ & & $2.07 \pm 0.07$ & $2.31 \pm 0.11$ \\
Stomach & $1,680 \pm 42$ & $1,564 \pm 28^{*}$ & & $5.33 \pm 0.10$ & $5.23 \pm 0.10$ \\
Duodenum & $471 \pm 25$ & $511 \pm 25$ & & $1.50 \pm 0.08$ & $1.71 \pm 0.07$ \\
Femur & $1,119 \pm 18$ & $1,020 \pm 13^{*}$ & & $3.56 \pm 0.06$ & $3.44 \pm 0.06$ \\
\hline
\end{tabular}

Values are means $\pm \mathrm{SE}$ for 18 control and 21 hindlimb-unweighted rats. $\mathrm{C}$, control group; $\mathrm{HU}, 15$-day hindlimb-unweighted group; EDL, extensor digitorum longus. ${ }^{*} \mathrm{HU}$ significantly different from control value, $P<0.05$.

as a post hoc test to estimate differences among means. To test for differences between control and 15-day $\mathrm{HU}$ mean values, a Student's unpaired two-tailed $t$ test was used. In all cases, the level of significance was set at $P<0.05$.

\section{RESULTS}

Tissue weights. Average values of tissue weights on which blood flow was measured are shown in Table 1. Tissue weights are expressed in both absolute units and normalized per body weight. In agreement with previous reports $(10,13,14)$ the postural, antigravity soleus muscle exhibited the greatest atrophy of the hindlimb muscles after HU. The soleus muscle weight was reduced $42 \%$ after HU [154 \pm 4 vs. $88 \pm 3$ (SE) $\mathrm{mg}$, and when normalized the average soleus muscle-to-body weight ratio was $38 \%$ lower than the control. The plantaris and gastrocnemius muscles also showed significant atrophy after HU. In addition, the deep knee extensor, vastus intermedius, was markedly smaller (tissue/body weight, $76 \%$ of control) after HU. The mean value of the vastus lateralis ( $\mathrm{mg}$ ) was significantly smaller in the HU group; however, when expressed per body weight, no significant difference existed. The adrenal medulla and cortex hypertrophied after 15 days of $\mathrm{HU}$ and when expressed per body weight were significantly larger than control (Table 1). In addition, both the stomach and femur weights (mg) were significantly reduced after $\mathrm{HU}$; however, when expressed per body weight the difference was not significant.

Distribution of blood flow. The absolute blood flows ( $\mathrm{ml} / \mathrm{min}$ ) and blood flows expressed per $100 \mathrm{~g}$ tissue weight $\left(\mathrm{ml} \cdot \mathrm{min}^{-1} \cdot 100 \mathrm{~g}^{-1}\right)$ during unweighting and quiescent standing are shown in Table 2, whereas blood flow during $\mathrm{PE}$ standing and running $(15 \mathrm{~m} / \mathrm{min})$ on the treadmill are presented in Table 3 . In both the control (unweighted acutely for $10 \mathrm{~min}$ ) and 15-day HU animals, flow to the soleus was significantly lower during unweighting compared with normal quiescent standing 
TABLE 2. Blood flows in organs and tissues during hindlimb unweighting and standing

\begin{tabular}{|c|c|c|c|c|}
\hline \multirow[b]{3}{*}{ Tissue } & \multicolumn{4}{|c|}{ Blood Flow } \\
\hline & \multicolumn{2}{|c|}{$\mathrm{ml} \cdot \min ^{-1} \cdot 10^{-2}$} & \multicolumn{2}{|c|}{$\mathrm{ml} \cdot \mathrm{min}^{-1} \cdot 100 \mathrm{~g}^{-1}$} \\
\hline & $\mathrm{C}$ & $\mathrm{HU}$ & $\mathrm{C}$ & $\mathrm{HU}$ \\
\hline Soleus & (11) & (11) & & \\
\hline Unwt & $\begin{array}{c}1.6 \pm 0.5 \\
(11)\end{array}$ & $\begin{array}{c}0.8 \pm 0.2 \\
(10)\end{array}$ & $8 \pm 1$ & $10 \pm 2$ \\
\hline Stand & $5.3 \pm 0.7 \dagger$ & $1.9 \pm 0.4 \dagger$ & $36 \pm 5 \dagger$ & $23 \pm 4 \dagger^{*}$ \\
\hline \multicolumn{5}{|l|}{ Plantaris } \\
\hline Unwt & $2.3 \pm 0.7$ & $2.7 \pm 0.8$ & $7 \pm 2$ & $12 \pm 3$ \\
\hline Stand & $2.3 \pm 0.4$ & $5.3 \pm 1.8$ & $7 \pm 1$ & $18 \pm 6$ \\
\hline \multicolumn{5}{|c|}{ Red gastrocnemius } \\
\hline Unwt & $0.8 \pm 0.2$ & $1.4 \pm 0.3$ & $8 \pm 3$ & $14 \pm 3$ \\
\hline Stand & $1.9 \pm 0.1$ & $3.5 \pm 1.2$ & $20 \pm 2$ & $38 \pm 15$ \\
\hline \multicolumn{5}{|c|}{ White gastrocnemius } \\
\hline Unwt & $1.3 \pm 0.3$ & $1.3 \pm 0.4$ & $7 \pm 1$ & $8 \pm 3$ \\
\hline Stand & $0.9 \pm 0.2$ & $0.7 \pm 0.3$ & $4 \pm 1$ & $5 \pm 2$ \\
\hline \multicolumn{5}{|c|}{ Mixed gastrocnemius } \\
\hline Unwt & $11.2 \pm 3.3$ & $10.9 \pm 1.7$ & $7 \pm 2$ & $9 \pm 2$ \\
\hline Stand & $10.9 \pm 2.6$ & $15.6 \pm 4.2$ & $8 \pm 2$ & $13 \pm 3$ \\
\hline \multicolumn{5}{|c|}{ Tibialis anterior red } \\
\hline Unwt & $3.0 \pm 1.1$ & $3.6 \pm 0.8$ & $27 \pm 9$ & $35 \pm 8$ \\
\hline Stand & $3.3 \pm 0.8$ & $1.5 \pm 0.3$ & $34 \pm 7$ & $14 \pm 3 \dagger^{*}$ \\
\hline \multicolumn{5}{|c|}{ Tibialis anterior white } \\
\hline Unwt & $7.5 \pm 2.0$ & $9.6 \pm 2.4$ & $13 \pm 4$ & $15 \pm 4$ \\
\hline Stand & $6.4 \pm 1.0$ & $4.1 \pm 0.6$ & $12 \pm 2$ & $8 \pm 1$ \\
\hline \multicolumn{5}{|l|}{ EDL } \\
\hline Unwt & $1.4 \pm 0.3$ & $1.6 \pm 0.6$ & $8 \pm 2$ & $10 \pm 4$ \\
\hline Stand & $1.3 \pm 0.3$ & $1.3 \pm 0.4$ & $8 \pm 1$ & $8 \pm 3$ \\
\hline \multicolumn{5}{|c|}{ Vastus intermedius } \\
\hline Unwt & $2.3 \pm 0.5$ & $1.8 \pm 0.5$ & $20 \pm 5$ & $21 \pm 5$ \\
\hline Stand & $4.0 \pm 0.8$ & $4.0 \pm 1.1$ & $37 \pm 7$ & $44 \pm 11$ \\
\hline \multicolumn{5}{|l|}{ Vastus medialis } \\
\hline Unwt & $7.4 \pm 1.8$ & $6.9 \pm 2.0$ & $13 \pm 3$ & $14 \pm 3$ \\
\hline Stand & $6.3 \pm 2.4$ & $6.0 \pm 1.3$ & $11 \pm 4$ & $14 \pm 3$ \\
\hline \multicolumn{5}{|c|}{ Vastus lateralis red } \\
\hline Unwt & $5.1 \pm 1.4$ & $2.5 \pm 0.6$ & $32 \pm 9$ & $17 \pm 4$ \\
\hline Stand & $4.3 \pm 1.1$ & $4.0 \pm 1.3$ & $28 \pm 7$ & $27 \pm 8$ \\
\hline Vastus laterali & & & & \\
\hline Unwt & $0.3 \pm 0.1$ & $1.6 \pm 0.5^{*}$ & $2 \pm 1$ & $8 \pm 2^{*}$ \\
\hline Stand & $2.5 \pm 2.1$ & $1.1 \pm 0.3$ & $2 \pm 1$ & $5 \pm 1$ \\
\hline Vastus laterali & & & & \\
\hline Unwt & $6.8 \pm 1.7$ & $7.9 \pm 1.8$ & $8 \pm 2$ & $11 \pm 3$ \\
\hline Stand & $4.4 \pm 1.1$ & $6.1 \pm 1.7$ & $6 \pm 1$ & $9 \pm 2$ \\
\hline Adrenal medu & & & & \\
\hline Unwt & $3.6 \pm 0.7$ & $4.3 \pm 0.7$ & $100 \pm 15$ & $105 \pm 21$ \\
\hline Stand & $3.0 \pm 0.5$ & $3.7 \pm 0.5$ & $90 \pm 12$ & $97 \pm 18$ \\
\hline Adrenal corter & & & & \\
\hline Unwt & $14.0 \pm 4.0$ & $21.0 \pm 6.0$ & $482 \pm 126$ & $683 \pm 168$ \\
\hline Stand & $13.0 \pm 3.0$ & $22.0 \pm 6.0$ & $471 \pm 98$ & $732 \pm 189$ \\
\hline Left kidney & & & & \\
\hline Unwt & $750.0 \pm 80.0$ & $650.0 \pm 60.0$ & $576 \pm 58$ & $488 \pm 45$ \\
\hline Stand & $800.0 \pm 61.0$ & $690.0 \pm 60.0$ & $611 \pm 46$ & $527 \pm 46$ \\
\hline Right kidney & & & & \\
\hline Unwt & $750.0 \pm 80.0$ & $680.0 \pm 60.0$ & $575 \pm 64$ & $510 \pm 49$ \\
\hline Stand & $800.0 \pm 58.0$ & $710.0 \pm 70.0$ & $623 \pm 44$ & $540 \pm 50$ \\
\hline Spleen & & & & \\
\hline Unwt & $80.0 \pm 27.0$ & $68.0 \pm 21.0$ & $124 \pm 42$ & $71 \pm 12$ \\
\hline Stand & $91.0 \pm 13.0$ & $91.0 \pm 21.0$ & $143 \pm 22$ & $123 \pm 23$ \\
\hline Stomach & & & & \\
\hline Unwt & $110.0 \pm 20.0$ & $110.0 \pm 20.0$ & $67 \pm 13$ & $71 \pm 12$ \\
\hline Stand & $130.0 \pm 23.0$ & $120.0 \pm 20.0$ & $80 \pm 15$ & $78 \pm 10$ \\
\hline Duodenum & & & & \\
\hline Unwt & $100.0 \pm 20.0$ & $100.0 \pm 20.0$ & $232 \pm 43$ & $217 \pm 46$ \\
\hline Stand & $100.0 \pm 13.0$ & $120.0 \pm 10.0$ & $215 \pm 25$ & $255 \pm 26$ \\
\hline Femur & & & & \\
\hline Unwt & $21.0 \pm 2.0$ & $18.0 \pm 3.0$ & $18 \pm 2$ & $17 \pm 3$ \\
\hline Stand & $23.0 \pm 2.0$ & $22.0 \pm 2.0$ & $20 \pm 1$ & $22 \pm 2$ \\
\hline
\end{tabular}

Values are means $\pm \mathrm{SE}$ for number of observations shown in parentheses. Unwt, 10-min acute and 15-day hindlimb unweighting for control and HU groups, respectively; Stand, 10-min standing after the unweighting in both groups. ${ }^{*} \mathrm{HU}$ significantly different from control values, $P<0.05 ; \dagger$ standing significantly different from unweighted values, $P<0.05$. (control, $8 \pm 1$ vs. $36 \pm 5 ; \mathrm{HU}, 10 \pm 2$ vs. $23 \pm 4 \mathrm{ml} \cdot \mathrm{min}^{-1}$. $\left.100 \mathrm{~g}^{-1}\right)$. The flow to the red portion of the tibialis anterior (TA), an ankle flexor muscle, was significantly greater during unweighting than while standing in the chronically unweighted animals $(35 \pm 8$ vs. $14 \pm 3$ $\mathrm{ml} \cdot \min ^{-1} \cdot 100 \mathrm{~g}^{-1}$ ). This increase in TA blood flow is likely due to plantar flexion of the foot and subsequent stretching of the TA, which occurs frequently during chronic HU. Also during unweighting, the flow to the white portion of the vastus lateralis was significantly greater in 15-day $\mathrm{HU}$ animals than in control animals ( $2 \pm 1$ vs. $8 \pm 2 \mathrm{ml} \cdot \min ^{-1} \cdot 100 \mathrm{~g}^{-1}$ ) (Table 2, Fig. 1). This was the only tissue that exhibited a significant difference between the two groups during unweighting.

During normal quiescent standing, the blood flow to the soleus was found to be significantly lower in the HU than in the control group ( $36 \pm 5 \mathrm{vs} .23 \pm 4 \mathrm{ml} \cdot \mathrm{min}^{-1} \cdot 100$ $\mathrm{g}^{-1}$; Fig. 2). Although the PE standing blood flow ( $\mathrm{ml} /$ $\mathrm{min}$ ) in the soleus was significantly lower than control soleus $(0.12 \pm 0.02$ vs. $0.05 \pm 0.01 \mathrm{ml} / \mathrm{min})$, flow in $\mathrm{ml} \cdot \mathrm{min}^{-1} \cdot 100 \mathrm{~g}^{-1}$ was not different between the two groups during unweighting, $\mathrm{PE}$, or $15 \mathrm{~m} / \mathrm{min}$ running (Fig. 2). As with the soleus, blood flow to the red portion of TA during standing was significantly lower $\left(34 \pm 7\right.$ vs. $14 \pm 3 \mathrm{ml} \cdot \mathrm{min}^{-1} \cdot 100 \mathrm{~g}^{-1}$ ) in 15 -day $\mathrm{HU}$ rats than in controls; and flows during unweighting, $\mathrm{PE}$, and $15 \mathrm{~m} / \mathrm{min}$ running were not different between groups (Fig. 3). Tissue blood flow in $\mathrm{ml} \cdot \mathrm{min}^{-1} \cdot 100 \mathrm{~g}^{-1}$ during $\mathrm{PE}$ standing was not different between groups except in the kidneys, where flow was significantly less in the $\mathrm{HU}$ group (Table 3; see Fig. 5).

Both groups showed an exercise-induced increase in hindlimb musculature blood flow. However, only the control group underwent a consistent decrease in blood flow to visceral organs (Table 3, Figs. 4 and 5). For example, exercise in control rats was associated with a marked drop in duodenum and kidney blood flow compared with the PE standing value, whereas flow to these two organs in HU animals was unaltered by exercise (Figs. 4 and 5 ).

Heart rate and blood pressure. Mean heart rate and arterial blood pressure values for control and HU animals during the four conditions are presented in Table 4 . In all four conditions mean heart rates were higher in the $\mathrm{HU}$ group, with significant differences observed during PE and exercise (Fig. 6). Mean arterial blood pressure did not differ between control and $\mathrm{HU}$ groups except during PE standing.

\section{DISCUSSION}

A major goal of this study was to characterize the distribution of blood flow between cage control and 15-day HU rats during unweighting, standing, and a bout of exercise. The important findings were 1) blood flow was significantly reduced to the postural antigravity soleus muscle during both acute (10-min) and chronic (15-day) HU compared with flow during standing; 2) after 15 days of $\mathrm{HU}$, blood flow to the soleus was significantly reduced during normal standing but not during a bout of exercise at the same absolute work load; and 3) 15 days of HU altered the redistribution of blood flow generally elicited by exercise. 
TABLE 3. Blood flows to organs and tissues during preexercise treadmill standing and treadmill running $(15 \mathrm{~m} / \mathrm{min})$

\begin{tabular}{|c|c|c|c|c|c|c|c|c|c|}
\hline \multirow[b]{3}{*}{ Tissue } & \multicolumn{4}{|c|}{ Blood Flow } & \multirow[b]{3}{*}{ Tissue } & \multicolumn{4}{|c|}{ Blood Flow } \\
\hline & \multicolumn{2}{|c|}{$\mathrm{ml} \cdot \min ^{-1} \cdot 10^{2}$} & \multicolumn{2}{|c|}{$\mathrm{ml} \cdot \mathrm{min}^{-1} \cdot 100 \mathrm{~g}^{-1}$} & & \multicolumn{2}{|c|}{$\mathrm{ml} \cdot \min { }^{1} \cdot 10^{-2}$} & \multicolumn{2}{|c|}{$\mathrm{ml} \cdot \min { }^{1} \cdot 100 \mathrm{~g}^{1}$} \\
\hline & $\mathrm{C}$ & $\mathrm{Hu}$ & C & $\mathrm{HL}$ & & C & $\mathrm{HU}$ & $\mathrm{C}$ & $\mathrm{HU}$ \\
\hline Soleus & (5) & $(6)$ & & & Vastus lateralis red & & & & \\
\hline PE & $\begin{array}{c}12.0 \pm 2.0 \\
(9)\end{array}$ & $\begin{array}{c}5.0 \pm 1.0^{*} \\
(7)\end{array}$ & $69 \pm 13$ & $55 \pm 11$ & $\begin{array}{l}\mathrm{PE} \\
15 \mathrm{~m} / \mathrm{min}\end{array}$ & $\begin{array}{l}12.0 \pm 3.0 \\
28.0 \pm 5.0\end{array}$ & $\begin{array}{l}13.0 \pm 3.0 \\
24.0 \pm 5.0\end{array}$ & $\begin{array}{r}83 \pm 21 \\
192 \pm 33\end{array}$ & $\begin{array}{l}107 \pm 33 \\
189 \pm 41\end{array}$ \\
\hline $\begin{array}{l}15 \mathrm{~m} / \mathrm{min} \\
\text { Plantaris }\end{array}$ & $14.0 \pm 2.0$ & $9.0 \pm 2.0$ & $85 \pm 8$ & $105 \pm 21$ & $\begin{array}{l}\text { Vastus lateralis } \\
\text { white }\end{array}$ & & & & \\
\hline PE & $8.7 \pm 1.8$ & $9.7 \pm 4.8$ & $22 \pm 5$ & $31 \pm \mathbf{1 6}$ & $\mathrm{PE}$ & $1.8 \pm 0.4$ & $2.2 \pm 0.9$ & $9 \pm 2$ & $9 \pm 4$ \\
\hline $15 \mathrm{~m} / \mathrm{min}$ & $26.0 \pm 6.0$ & $17.0 \pm 3.0$ & $69+15$ & $58 \pm 9$ & $15 \mathrm{~m} / \mathrm{min}$ & $1.9 \pm 0.6$ & $3.3+0.9$ & $10 \pm 3$ & $16 \pm 4$ \\
\hline $\begin{array}{l}\text { Red gastrocnemius } \\
\text { PE }\end{array}$ & $9.0 \pm 2.0$ & $7.0 \pm 2.0$ & $94 \pm 24$ & $76 \pm 22$ & $\begin{array}{l}\text { Vastus lateralis } \\
\text { mixed }\end{array}$ & & & & \\
\hline $15 \mathrm{~m} / \mathrm{min}$ & $15.0 \pm 3.0$ & $10.0 \pm 1.0$ & $156 \pm 25$ & $108 \pm 12$ & $\mathrm{PE}$ & $23.0 \pm 5.0$ & $17.0 \pm 7.0$ & $25 \pm 5$ & $21 \pm 9$ \\
\hline $\begin{array}{l}\text { White } \\
\text { gastrocnemius }\end{array}$ & & & & & $\begin{array}{l}15 \mathrm{~m} / \mathrm{min} \\
\text { Adrenal medulla }\end{array}$ & $57.0 \pm 17.0$ & $42.0 \pm 8.0$ & $63 \pm 18$ & $56 \pm 10$ \\
\hline $\mathrm{PE}$ & $0.8 \pm 0.2$ & $1.0 \pm 0.3$ & $5 \pm 1$ & $7 \pm 2$ & PE & $4.7 \pm 1.0$ & $6.7 \pm 1.0$ & $131 \pm 36$ & $197 \pm 51$ \\
\hline $15 \mathrm{~m} / \mathrm{min}$ & $1.9 \pm 0.4$ & $3.3 \pm 1.0$ & $10 \pm 2$ & $26 \pm 8$ & $15 \mathrm{~m} / \mathrm{min}$ & $3.6 \pm 1.0$ & $5.6 \pm 2.8$ & $102 \pm 29$ & $190 \pm 107$ \\
\hline Mixed & & & & & Adrenal cortex & & & & \\
\hline gastrocnemius & & & & & PE & $18.0 \pm 7.0$ & $40.0 \pm 16.0$ & $898 \pm 336$ & $1479 \pm 425$ \\
\hline $\mathrm{PE}$ & $34.0 \pm 7.0$ & $30.0 \pm 18.0$ & $21 \pm 4$ & $23 \pm 10$ & $15 \mathrm{~m} / \mathrm{min}$ & $19.0 \pm 7.0$ & $17.0 \pm 3.0$ & $963 \pm 415$ & $758 \pm 219$ \\
\hline $15 \mathrm{~m} / \mathrm{min}$ & $88.0 \pm 21.0$ & $87.0 \pm 16.0$ & $57 \pm 14$ & $70 \pm 11$ & Left kidney & & & & \\
\hline Tibialis anterior red & & & & & $\mathrm{PE}$ & $1,170.0 \pm 90.0$ & $740.0 \pm 120.0^{*}$ & $944 \pm 9: 3$ & $556 \pm 84^{*}$ \\
\hline $\mathrm{PE}$ & $5.7 \pm 2.6$ & $3.1 \pm 2.1$ & $46 \pm 15$ & $31 \pm 22$ & $15 \mathrm{~m} / \mathrm{min}$ & $520.0 \pm 80.0$ & $640.0 \pm 110.0$ & $416 \pm 71$ & $493 \pm 87$ \\
\hline $15 \mathrm{~m} / \mathrm{min}$ & $16.0 \pm 3.0$ & $9.0 \pm 2.0$ & $159 \pm 31$ & $95 \pm 17$ & Right kidney & & & & \\
\hline $\begin{array}{l}\text { Tibialis anterior } \\
\text { white }\end{array}$ & & & & & $\begin{array}{l}\mathrm{PE} \\
15 \mathrm{~m} / \mathrm{min}\end{array}$ & $\begin{array}{c}1,220.0 \pm 110.0 \\
530.0 \pm 90.0\end{array}$ & $\begin{array}{l}750.0 \pm 90.0^{*} \\
600.0 \pm 110.0\end{array}$ & $\begin{array}{l}974 \pm 92 \\
415 \pm 75\end{array}$ & $\begin{array}{l}556 \pm 66^{*} \\
469 \pm 92\end{array}$ \\
\hline $\mathrm{PE}$ & $13.0 \pm 4.0$ & $14.0 \pm 10.0$ & $20 \pm 6$ & $23 \pm 16$ & Spleen & & & & \\
\hline $15 \mathrm{~m} / \mathrm{min}$ & $27.0 \pm 5.0$ & $30.0 \pm 6.0$ & $45 \pm 9$ & $55 \pm 10$ & $\mathrm{PE}$ & $230.0 \pm 40.0$ & $130.0 \pm 40.0$ & $352 \pm 72$ & $207 \pm 62$ \\
\hline EDL & & & & & $15 \mathrm{~m} / \mathrm{min}$ & $76.0 \pm 7.0$ & $28.0 \pm 3.0$ & $104 \pm 41$ & $45 \pm 11$ \\
\hline PE & $3.7 \pm 1.2$ & $1.4 \pm 0.6$ & $20 \pm 7$ & $8 \pm 4$ & Stomach & & & & \\
\hline $15 \mathrm{~m} / \mathrm{min}$ & $3.9 \pm 0.7$ & $5.1 \pm 1.5$ & $22 \pm 4$ & $30 \pm 8$ & PE & $340.0 \pm 60.0$ & $190.0 \pm 70.0$ & $185 \pm 28$ & $122 \pm 42$ \\
\hline Vastus intermedius & & & & & $15 \mathrm{~m} / \mathrm{min}$ & $72.0 \pm 13.0$ & $75.0 \pm 16.0$ & $41 \pm 7$ & $49 \pm 9$ \\
\hline $\mathrm{PE}$ & $19.0 \pm 5.0$ & $3.9 \pm 1.0^{*}$ & $125 \pm 33$ & $47 \pm 13$ & Duodenum & & & & \\
\hline $15 \mathrm{~m} / \mathrm{min}$ & $22.0 \pm 4.0$ & $12.0 \pm 3.0$ & $160 \pm 29$ & $134 \pm 29$ & PE & $220.0 \pm 50.0$ & $220.0 \pm 50.0$ & $390 \pm 76$ & $341 \pm 49$ \\
\hline Vastus medialis & & & & & $15 \mathrm{~m} / \mathrm{min}$ & $41.0 \pm 8.0$ & $150.0 \pm 30.0^{*}$ & $79 \pm 10$ & $285 \pm 43^{*}$ \\
\hline $\mathrm{PE}$ & $17.0 \pm 4.0$ & $7.2 \pm 3.0$ & $34 \pm 10$ & $17 \pm 7$ & Femur & & & & \\
\hline $15 \mathrm{~m} / \mathrm{min}$ & $45.0 \pm 15.0$ & $32.0 \pm 5.0$ & $90 \pm 30$ & $73 \pm 12$ & $\mathrm{PE}$ & $26.0 \pm 7.0$ & $21.0 \pm 6.0$ & $26 \pm 6$ & $21 \pm 6$ \\
\hline & & & & & $15 \mathrm{~m} / \mathrm{min}$ & $18.0 \pm 3.0$ & $20.0 \pm 5.0$ & $17 \pm 2$ & $21 \pm 4$ \\
\hline
\end{tabular}

Values are means $\pm \mathrm{SE}$ for number of observations shown in parentheses. PE, preexercise. ${ }^{*}$ HU significantly different from cont rol values, $P<$ 0.05

Two widely used models simulating weightlessness, HU and HI, have been documented to effect soleus muscle structure, function, and metabolism. Witzmann et al. (37) found 42 days of $\mathrm{HI}$ to increase the rate of fatigue induced by electrode stimulation of the in situ soleus. Additionally, the atrophied soleus exhibited a greater glycogen and ATP usage and higher lactate production than did the control muscle (37). It is also known that HU elicits an elevated glycogen content in the soleus (17). Recent single fiber data have found 2 wk of $\mathrm{HU}$ to increase soleus muscle fiber glycogen, ATP, and creatine phosphate (18). These findings suggest an atrophied-induced shift in metabolism to a higher reliance on carbohydrate and anaerobic (glycolytic) energy production. This observed shift occurs despite an increased oxidative capacity (per fiber weight) after both $\mathrm{HU}$ and $\mathrm{HI}(12,16)$. These observations led us to hypothesize that the increased reliance of the atrophied soleus on anaerobic energy production might result from a reduced muscle blood flow, leading to a low $\mathrm{O}_{2}$ and free fatty acid (FFA) delivery to exercising hindlimb muscles.

Both acute (10-min) and chronic (15-day) HU decreased blood flow to the soleus, a predominantly slow- twitch oxidative (SO) muscle (3). This was likely due to a reduction in muscular activity and a corresponding decrease in the release of vasodilatory metabolites in the soleus, which is near maximally active during postural maintenance $(19,33)$. In other muscles composed predominantly of fast-twitch oxidative-glycolytic (FOG) and fast-twitch glycolytic (FG) fibers, blood flow did not change with acute unweighting in control animals, presumably because of insignificant changes in muscular activity, because these muscle types are relatively inactive during standing $(19,33)$. However, blood flow to predominantly FG muscle (i.e., white regions of the gastrocnemius and vastus lateralis) after chronic unweighting was $157 \%$ higher than that of acutely unweighted muscle (Fig. 7). This may mechanistically be due to a decreased responsiveness to sympathetic activation and, subsequently, a decreased vascular tone and increased blood flow. Overton and Tipton (29) observed an attenuated mesenteric artery pressor response to exogenous sympathomimetic agents after 9 days of $\mathrm{HU}$; chronic unweighting may produce a prolonged stimulus to sympathetic drive $(11,29)$, as suggested by the observed hypertrophy of the adrenal glands (Table 1), resulting in a 


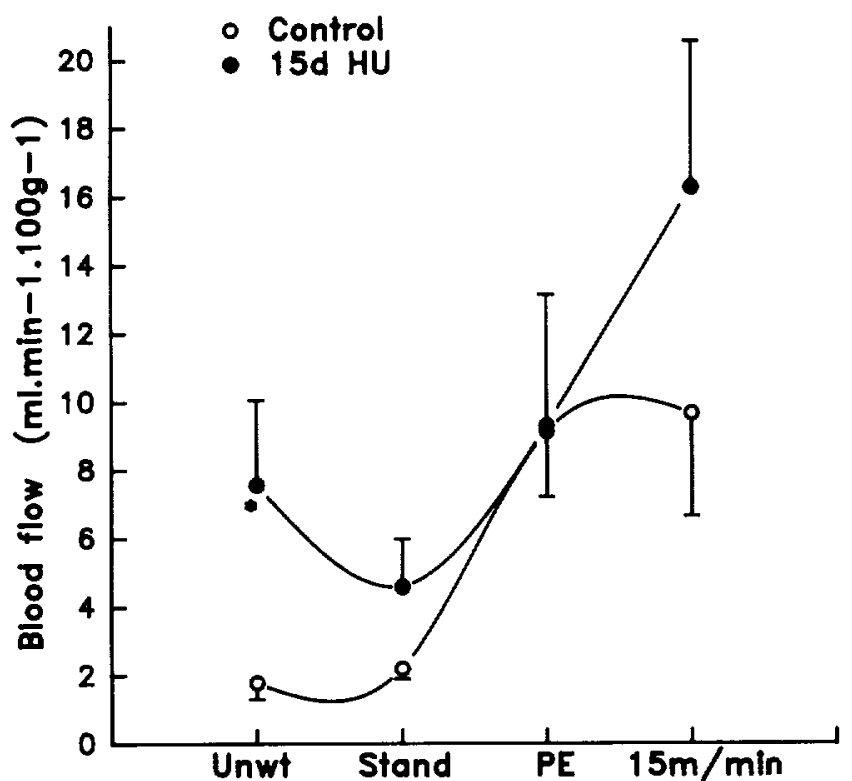

FIG. 1. White vastus lateralis blood flow (mean $\pm \mathrm{SE}$ ) during 4 conditions. Unwt, acute 10-min hindlimb unweighting (HU) for control group and after 15 days of $\mathrm{HU}$ for the HU group; Stand, normal standing; $\mathrm{PE}$, preexercise standing on treadmill; $15 \mathrm{~m} / \mathrm{min}$, treadmill run ning at $15 \mathrm{~m} / \mathrm{min} .{ }^{*} 15$-day $\mathrm{HU}$ value significantly different from control value, $P<0.05$.

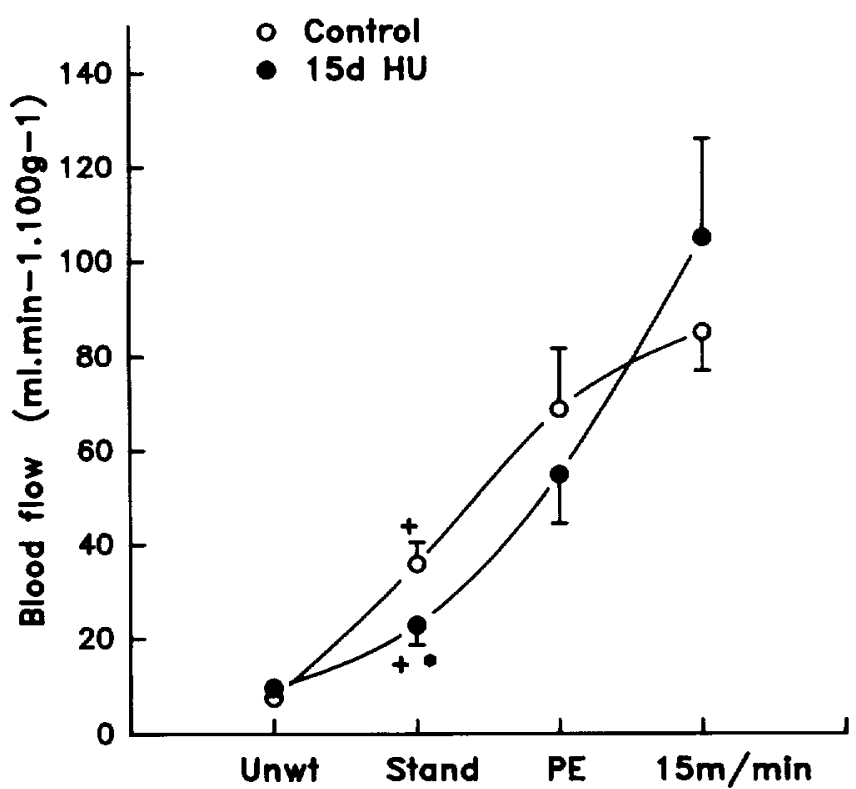

FIG. 2. Soleus muscle blood flow (mean $\pm \mathrm{SE}$ ) during 4 conditions. $x$-Axis abbreviations, same as Fig. 1. †Standing blood flow significantly different from unweighted value, $P<0.05$. ${ }^{*} 15$-day $\mathrm{HU}$ value significantly different from control value, $P<0.05$.

downregulation of $\alpha$-receptor number and/or sensitivity (38). In the rat, muscle composed primarily of FG fibers has the potential for greater control of vascular tone by the sympathetic nervous system than that composed of SO or FOG fibers $(9,21)$. Thus a reduction in sympathetically mediated vascular tone would be expected to primarily increase blood flow to muscle composed of FG fibers.

After 15 days of $\mathrm{HU}$, blood flow to the soleus was significantly reduced $\left(23 \pm 4\right.$ vs. $\left.36 \pm 5 \mathrm{ml} \cdot \mathrm{min}^{-1} \cdot 100 \mathrm{~g}^{-1}\right)$

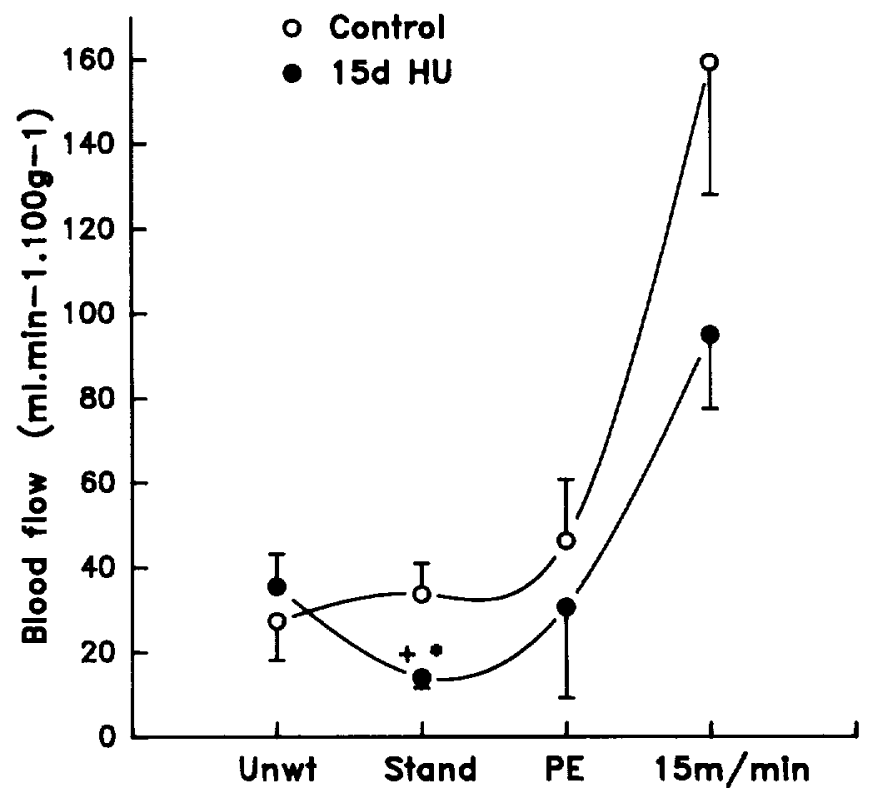

FIG. 3. Blood flows (mean $\pm \mathrm{SE}$ ) to red region of tibialis anterior during 4 conditions. $x$-Axis abbreviations, same as Fig. $1 .{ }^{*} 15$-day HU value significantly different from control, $P<0.05$.

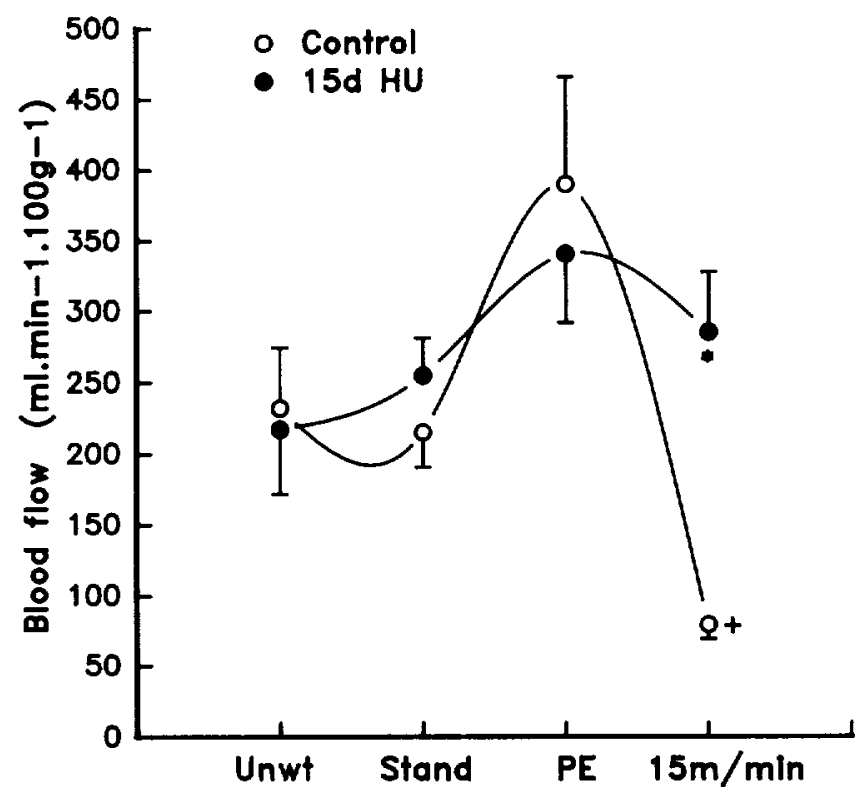

FIG. 4. Duodenum blood flow (mean $\pm \mathrm{SE}$ ) during 4 conditions. $x$ Axis abbreviations, same as Fig. $1 .+15 \mathrm{~m} / \mathrm{min}$ blood flow significantly different from $\mathrm{PE}$ value, $P<0.05$. * 15 -day $\mathrm{HU}$ value significantly different from control, $P<0.05$.

during quiescent standing. This is in agreement with LeBlanc et al. (23), who observed a decreased percent uptake of microspheres by the soleus after 14-90 days of HU. However, soleus muscle blood flow measured while the rats of the second study were standing on the treadmill (Table 3) was not different between controls and 15-day HU animals (control $69 \pm 3$; HU $55 \pm 11$ ). Two factors may account for the discrepancy in the standing soleus muscle flows between the two studies. First, both control and 15-day HU animals in the first study were behaviorally very quiescent after being lowered from an unweighted to standing position (on laboratory bench). The relaxed posture maintained by these animals may 


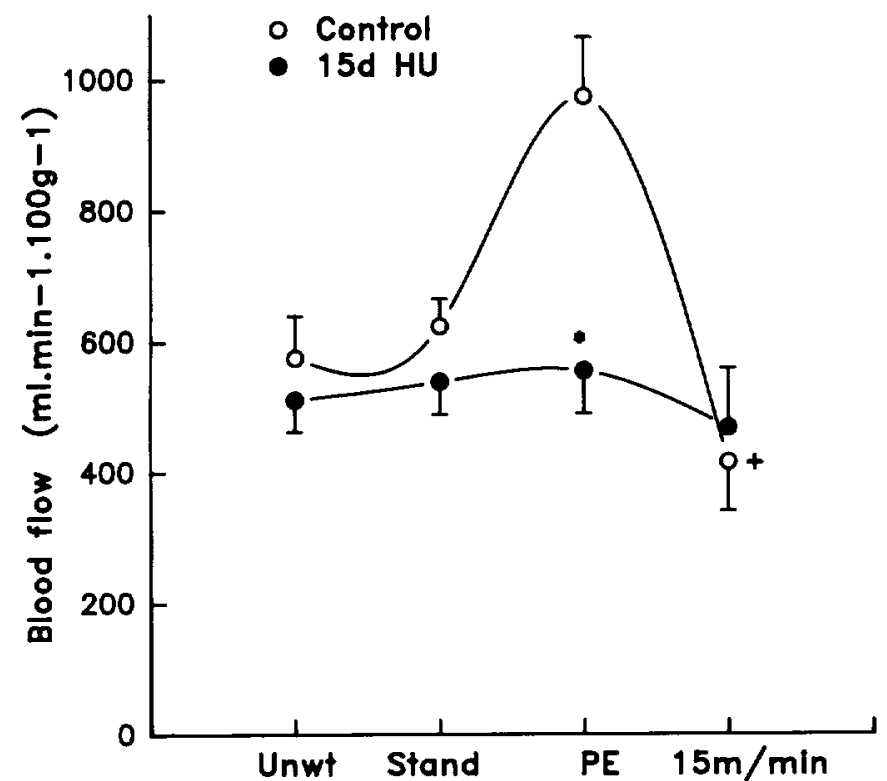

FIG. 5. Blood flow (mean $\pm \mathrm{SE}$ ) to right kidney during 4 conditions $x$-Axis abbreviations, same as Fig. $1 .+15 \mathrm{~m} / \mathrm{min}$ blood flow significantly different from $\mathrm{PE}$ value, $P<0.05 .{ }^{*} 15$-day $\mathrm{HU}$ value significantly dif. ferent from control, $P<0.05$.

TABLE 4. Heart rate and blood pressure

\begin{tabular}{|c|c|c|c|c|}
\hline \multirow[b]{2}{*}{ Condition } & \multicolumn{2}{|c|}{ Heart Rate, beats/min } & \multicolumn{2}{|c|}{$\begin{array}{l}\text { Mean Arterial Blood } \\
\text { Pressure, } \mathrm{mmHg}\end{array}$} \\
\hline & $\mathrm{C}$ & $\mathrm{HU}$ & $\mathrm{C}$ & $\mathrm{HU}$ \\
\hline Unweighted & $433 \pm 14(11)$ & $460 \pm 18(12)$ & $138 \pm 2(7)$ & $138 \pm 4(9)$ \\
\hline Standing & $394 \pm 10(11)$ & $429 \pm 14(12)$ & $127 \pm 3(8)$ & $127 \pm 4(10)$ \\
\hline Preexercise & $428 \pm 17(5)$ & $509 \pm 17^{*}(6)$ & $117 \pm 4(5)$ & $135 \pm 5 *(6)$ \\
\hline $\begin{array}{l}\text { Exercise } \\
\qquad(15 \mathrm{~m} / \mathrm{min})\end{array}$ & $478 \pm 16(9)$ & $583 \pm 11^{*}(8)$ & $138 \pm 6(9)$ & $143 \pm 4(9)$ \\
\hline
\end{tabular}

Values are means \pm SE for number of observations shown in parentheses. ${ }^{*} \mathrm{HU}$ significantly different from control values, $P<0.05$.

account for the low flow in the postural antigravity soleus muscle compared with the other standing/preexercise flows in this and other studies $(7,19)$. Additionally, reposturing to relieve the atrophied soleus muscle from as much weight bearing as possible may have caused the lower soleus muscle blood flow in the 15-day HU animals of the first study. Second, the higher standing/preexercise soleus muscle blood flows in the second study may be due to an anticipatory response, because flow was measured after the animals were placed on the treadmill and anticipated an ensuing bout of exercise. Previous studies have shown that rats conditioned (1) or trained (2) to run on a treadmill have increased standing flows in several muscles. Thus standing blood flows may have been increased by placing the animals of the second study, which had been previously conditioned to treadmill running, on the treadmill. The higher heart rate of these rats, which is indicative of anticipation to exercise $(1,2)$, supports this hypothesis.

There were no significant differences between chronically unweighted and control animal muscle blood flows per $100 \mathrm{~g}$ tissue during treadmill running at $15 \mathrm{~m} / \mathrm{min}$. Additionally, our findings show patterns of muscle fiber recruitment during low-intensity exercise similar to

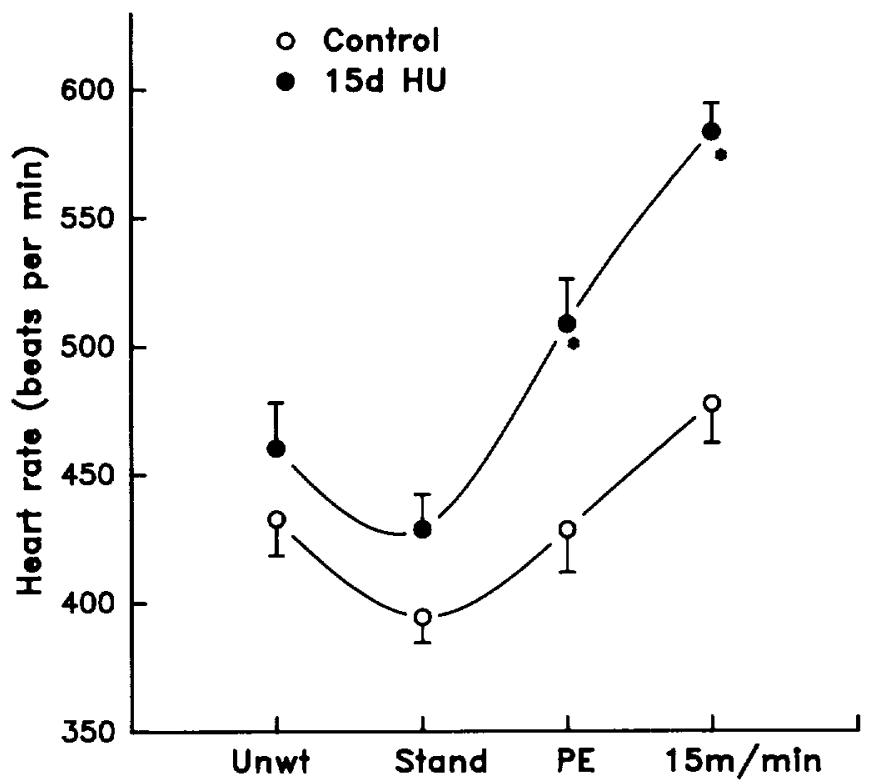

FIG. 6 . Heart rate (mean $\pm \mathrm{SE}$ ) during 4 conditions. $x$-Axis abbreviations, same as Fig. $1 .{ }^{*} 15$-day HU significantly different from control values, $P<0.05$.

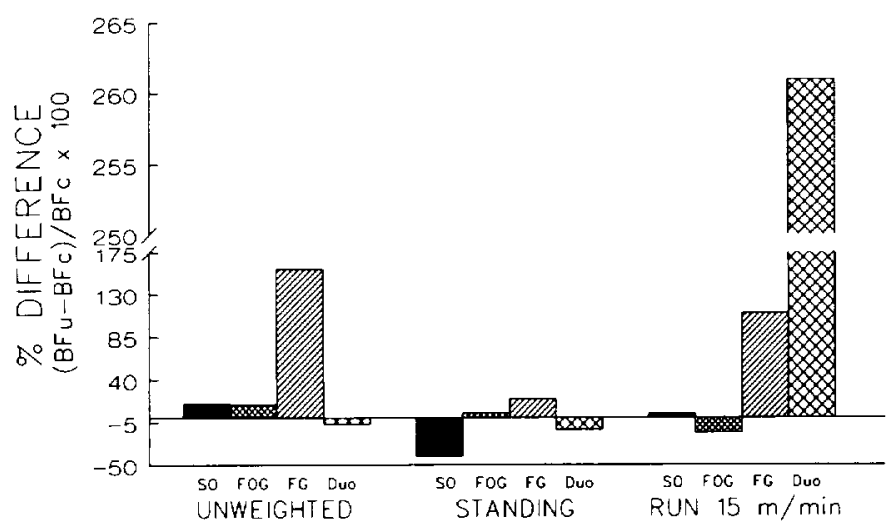

FIG. 7 . Percent differences in blood flows (BF; $\mathrm{ml} \cdot \mathrm{min}^{-1} \cdot 100 \mathrm{~g}^{-1}$ ) between chronically unweighted (u) and control (c) rats for extensor muscles composed primarily of slow-twitch oxidative (SO, soleus and vastus intermedius muscles), fast-twitch oxidative-glycolytic (FOG, red portions of gastrocnemius and vastus lateralis muscles), and fasttwitch glycolytic (FG, white portions of gastrocnemius and vastus lateralis muscles) fibers and duodenum (Duo) during hindlimb unweighting, preexercise standing, and exercise.

those of Laughlin and Armstrong (19), who demonstrated that exercise-induced increases in hindlimb blood flow are preferentially directed to muscles primarily composed of FOG fibers. However, chronic unweighting did influence the distribution of cardiac output during treadmill running. During low-intensity exercise, oxidative (SO and FOG) muscle blood flow increases and glycolytic (FG) muscle and visceral flows decrease (19). After chronic unweighting, duodenum (Fig. 4) and kidney (Fig. 5) blood flows remained elevated with exercise. As mentioned above, this failure to redistribute the cardiac output to the active muscle may be due to a downregulation of $\alpha$-receptors and a subsequent attenuation in responsiveness to sympathetic activation (29). Although less apparent, unweighting may also result in a decrease in sympathetically mediated vascular tone in muscle composed of FG fibers during exercise (Fig. 7). This could 
explain the 60 and $160 \%$ greater blood flow observed in the white portions of vastus lateralis and gastrocnemius muscles, respectively, after chronic unweighting.

Although cardiac output was not measured in this study, a few simple calculations using regional flows can be made to estimate the effects of hypodynamia on this variable during exercise. Using estimates of total muscle [39\% total body mass (8)], bone [6\% total body mass (8)], and visceral [ $8 \%$ total body mass (8)] mass multiplied by the ratio of summed tissue blood flows $(\mathrm{ml} / \mathrm{min}$, Table 3$)$ and summed tissue masses (Table 1) as representative of average muscle, bone or visceral blood flow per gram of tissue, then total flows to these tissues, can be deduced. Muscle, bone, and visceral blood flow would be 96,3 , and $58 \mathrm{ml} / \mathrm{min}$, respectively, in control rats and 85,4 , and 67 $\mathrm{ml}$, respectively, in chronically unweighted rats during exercise. Total blood flow to these three body compartments would be $157 \mathrm{ml} / \mathrm{min}$ in control and $156 \mathrm{ml} / \mathrm{min}$ in unweighted animals. These calculations are only estimates and assume that relative body compositions are similar, but they suggest that cardiac output at the same absolute exercise intensity is unaffected by chronic unweighting if blood flows to other body compartments, e.g., skin and fat, are not different. Saltin and co-workers (32) have previously reported that cardiac output in humans during submaximal exercise does not change after 20 days of bed rest.

The above calculations indicate that total muscle blood flow $(\mathrm{ml} / \mathrm{min})$ during exercise was less after prolonged unweighting. There were no significant differences in individual muscle flows during exercise between control and chronically unweighted animals. However, of the 13 muscles and muscle parts sampled, 9 had absolute decreases in flow and 4, all having high populations of FG fibers, had absolute increases in blood flow. The sum effect of small insignificant changes in blood flow to individual muscles can result in significant changes in total muscle flow (8). The presumed reduction in total muscle blood flow could quantitatively be accounted for by the lack of a reduction in visceral flows. The apparent inability to redistribute blood flow away from inactive to active tissue could have significant adverse consequences on total body aerobic metabolism and exercise performance. For example, Overton et al. (30) reported that maximal oxygen consumption $\left(\mathrm{V}_{\mathrm{V}_{\text {max }}}\right)$ is reduced after $6-9$ days of head-down or horizontal unweighting. If visceral flows continue to remain elevated during high-intensity exercise and a lower percentage of maximal cardiac output is directed to active muscle, then the loss of ability to redistribute cardiac output after chronic unweighting may have a causal role in reducing $\dot{\mathrm{VO}}_{2 \text { max }}$.

If $\mathrm{VO}_{2 \max }$ were reduced in our rats, then the chronically unweighted rats in this study were exercising at a higher relative $\mathrm{O}_{2}$ consumption during the $15 \mathrm{~m} / \mathrm{min}$ exercise bout. Because soleus muscle blood flows between groups were the same during exercise at these potentially different relative work loads, the atrophied soleus muscle blood flow might have been lower than the control value had both groups been exercising at the same relative work load. Additionally, the apparent lack of control by the sympathetic nervous system to direct cardiac output becomes even more impressive, because visceral blood flow is inversely related to exercise intensity (19).

Twenty days of deconditioning bed rest in humans induced tachycardia at rest and during submaximal exercise (32). Prolonged HU similarly induced PE standing and exercise tachycardia in this study, but this was in contrast to another unweighted rat study of shorter duration (30). Exercise training also has been shown to induce bradycardia during exercise in rats $(2,15)$. These observations indicate that the chronotropic state of the heart adapts to both chronic increases and decreases of activity in rats.

Rat HU provides a model for muscular hypodynamia. The effects of prolonged reductions in muscular weightbearing and locomotory activity on hindlimb vascular morphology and tone might be the inverse of those after chronic increases in contractile activity. For example, exercise training increased rat skeletal muscle vascular capacity for flow $(22,24)$, a response that was likely in part caused by an increased capillarity (25). Despite an increased vascular capacity after training, total hindlimb blood flow did not change during treadmill running (2). However, there was a marked redistribution of flow within the hindlimb, with the trained animals having higher blood flows to high-oxidative (SO and FOG) muscles and lower flows to low-oxidative (FG) muscle (2). All muscle types had elevated succinate dehydrogenase activities after training, so it appears that the vascular adaptations to training were fiber type specific; i.e., vessels in high- and low-oxidative muscles adapted with a decreased and increased vascular tone, respectively. Chronic reductions in activity via $\mathrm{HU}$ induced a decreased capillary-to-fiber ratio in soleus muscle (10). If the number of fibers in soleus muscle did not change (35), then this would indicate that a reduction in the absolute number of capillaries, and presumably a reduction in the absolute vascular transport capacity, occurred. However, capillary density increased with unweighting (10), indicating the decrease in fiber mass was greater than the decrease in capillary number. Therefore vascular capacity for flow relative to muscle mass may be elevated after hypodynamia. Although the effects of prolonged inactivity on vascular transport capacity have not been directly tested, the results of this study support the contention that HU does not adversely affect blood flow per unit mass of muscle during submaximal exercise. However, as with exercise training, it appears that adaptations in the control of vascular tone occurred with chronic unweighting, and the mechanisms involved in this adaptation process merit further investigation.

The observation that soleus blood flow during exercise was not different between groups leads us to reject the hypothesis that a reduced blood flow caused the elevated glycogen depletion and lactate production observed with contractile activity in atrophy soleus muscle (37). The question remains as to what triggers the altered substrate metabolism. One possibility is a $\mathrm{HU}$-induced decrease in the responsiveness to sympathetic activation (29). As previously stated, this is consistent with our finding that blood flow to muscle composed of predominantly FG fibers is elevated during unweighting and flow to the 
duodenum and kidneys remained elevated during exercise in $\mathrm{HU}$ rats. A reduced responsiveness to sympathetic drive could inhibit the work-induced mobilization of FFA, decreasing blood FFA and thus their delivery, uptake, and oxidation by skeletal muscle. This hypothesis is supported by the observed hypertrophy of the adrenal medulla and cortex after 15 days of HU. A second possibility for the greater reliance of hindlimb muscles on glycolytic metabolism after $\mathrm{HU}$ is a reduced blood $\mathrm{O}_{2}$-carrying capacity. Reductions in plasma volume and erythrocyte mass have been reported after unweighting $(6,11$, 30). The relative importance of these two possibilities in altering the exercise metabolism of the atrophied soleus awaits an analysis of blood gases and FFA content during exercise of chronically unweighted animals.

In conclusion, on unweighting, blood flow to the soleus is significantly reduced. After 15 days unweighting, flow to the soleus is depressed during normal standing but not during a bout of treadmill running. Hence, the increased dependence of atrophied soleus on glycogen and anaerobic energy production during contractile activity cannot be accounted for by an attenuated blood flow. The fact that chronic $\mathrm{HU}$ blunts the redistribution of visceral blood flow during exercise suggests a reduced cellular response to catecholamines. Consequently, with the onset of exercise, the epinephrine-mediated increase in plasma FFA might be inhibited, producing an increased dependence on muscle glycogen in the atrophied soleus.

We thank Barbara DeNoyer for help in the preparation of the manuscript and Dr. Robert B. Armstrong for advice in the design of the blood flow experiments.

This research was supported by National Aeronautics and Space Administration Grant NAG 2-212.

Address for reprint requests: R. H. Fitts, Biology Dept., Marquette University, Milwaukee, WI 53233.

Received 19 December 1990; accepted in final form 16 December 1991.

\section{REFERENCES}

1. Armstrong, R. B., D. A. Hayes, and M. D. Delp. Blood flow distribution in rat muscles during preexercise anticipatory response. J. Appl. Physiol. 67: 1855-1861, 1989.

2. ARMSTrong, R. B., and M. H. LaUghlin. Exercise blood flow patterns within and among rat muscles after training. Am. J. Physiol. 246 (Heart Circ. Physiol. 15): H59-H68, 1984.

3. Armstrong, R. B., and R. O. Phelps. Muscle fiber type composition of rat hindlimb. Am. J. Anat. 171: 259-272, 1984.

4. Chiver, C. C., AND I. J. Kopin. Hyperresponsivity in spontaneously hypertensive rat to indirect measurement of blood pressure. Am. J. Physiol. 234 (Heart Circ. Physiol. 3): H690-H695, 1978.

5. Convertino, V. A. Physiological adaptations to weightlessness: effects on exercise and work performance. Exercise Sports Sci. Rev. 18: $119-168,1990$

6. Deavers, D. R., X. J. Musacchia, and G. A. Meininger. Model for antiorthostatic hypokinesia: head down tilt effects on water and salt excretion. J. Appl. Physiol. 49: 576-582, 1980.

7. DELP, M. D., AND R. B. ARmstrong. Blood flow in normal and denervated muscle during exercise in conscious rats. Am. J. Physiol. 255 (Heart Circ. Physiol. 24): H1509-H1515, 1988.

8. Delp, M. D., R. O. ManNing, J. V. Bruckner, and R. B. ARMSTRONG. Distribution of cardiac output during diurnal changes of activity in rats. Am. J. Physiol. 261 (Heart Circ. Physiol. 30): H1487-H149;, 1991.

9. Delp, M. D., J. A. Warren, AND R. B. Armstrong. Sympathetic control of vascular tone in muscles of different fiber types $(\mathrm{Ab}$. stract). Med. Sci. Sports Exercise 22: S105, 1990.
10. Desplanches, D., M. H. Mayet, B. Sempore, and R. FlanDRoIs. Structural and functional responses to prolonged hindlimb suspension in rat muscle. $J$. Appl. Phvsiol. 63: 558-563, 1987.

11. Dunn, C. D. R., P. C. Johnson, And R. D. Lange. Regulation of hematopoiesis in rats exposed to antiorthostatic hypokinetic/hypodynamia. II. Mechanisms of the "anemia." Aviat. Space Environ. Med. 57: 36-44, 1986.

12. FitTs, R. H., C. J. Brimmer, A. Heywood-Cooksey, AND R. J. Timmerman. Single muscle fiber enzyme shifts with hindlimb suspension and immobilization. Am. J. Physiol. 256 (Cell Physiol. 25): C1082-C1091, 1989.

13. FitTs, R. H., J. M. Metzger, D. A. Ril.ey, And B. R. Unsworth. Models of disuse: a comparison of hindlimb suspension and immobilization. J. Appl. Physiol. 60: 1946-1953, 1986.

14. Gardetto, P. R., J. M. Schluter, and R. H. FitTs. Contractile function of single muscle fibers after hindlimb suspension. $J$. Appl. Physiol. 66: 2739-2749, 1989.

15. Glefison, T. T., W. J. Mullin, and K. M. Baldwin. Cardiovascular responses to treadmill exercise in rats: effects of training. $J$. Appl. Physiol. 54: 789-793, 1983.

16. HaUSCHKa, E. O., R. R. RoY, AND V. R. Edger'Ton. Size and metabolic properties of single muscle fibers in rat soleus after hindlimb suspension. J. Appl. Physiol. 62: 2338-2347, 1987

17. HENRIKSEN, E. J., M. E. Tischler, AND D. G. Johnson. Increased response to insulin of glucose metabolism in the 6 -day unloaded rat soleus muscle. J. Biol. Chem. 261: 10707-10712, 1986.

18. HeYwood-Cooksey, A., AND R. H. FitTs. The effect of hindlimb suspension on the substrate protile of fast and slow skeletal muscle fiber types (Abstract). Med. Sci. Sports Exercise 23: S129, 1991

19. Laughlin, M. H., And R. B. ARmstrong. Muscular blood flow distribution patterns as a function of running speeds in rats. $A m . J$. Physiol. 243 (Heart Circ. Physiol. 12): H296-H306, 1982.

20. Laughlin, M. H., AND R. B. ARMSTRONG. Adrenoceptor effects on rat muscle blood flow during treadmill exercise. $J$. Appl. Physiol. 62: $1465-1472,1987$

21. Laughlin, M. H., R. B. Armstrong, J. White, AND K. Rouk. A method for using microspheres to measure muscle blood flow in exercising rats. J. Appl. Physiol. 52: 1629-1635, 1982.

22. LAUGHLIN, M. H., AND J. RIPPERGER. Vascular transport capacity of hindlimb muscles of exercise-trained rats. J. Appl. Physiol. 62: $438-443,1987$

23. LeBlanc, A., C. Marsh, H. Evans, P. Johnson, V. Schneider, AND S. JHINGRAN. Bone and muscle atrophy with suspension of the rat. J. Appl. Physiol. 58: 1669-1675, 1985.

24. MACKIE, B. G., AND R. L. TerJuNG. Influence of training on blood flow to different skeletal muscle fiber types. J. Appl. Physiol. 55: $1072-1078,1983$.

25. MaI, J. V., V. R. EdgerTon, AND R. J. BarNaRd. Capillarity of red, white, and intermediate muscle fibers in trained and untrained guinea pigs. Experientia Basel 26: 1222-1223, 1970.

26. Martin, T. P., V. R. Edgerton, and R. E. Grindeland. Influence of spaceflight on rat skeletal muscle. J. Appl. Physiol 65: 2318-2325, 1988

27. MCDonald, K. S., M. D. DelP, and R. H. FiTTS. Relationship between contractile function and blood flow in the soleus following hindlimb suspension (Abstract). Med. Sci. Sport. Exercise 23: $\$ 24$, 1991 .

28. Miu, B., T. P. Martin, R. R. Roy, V. Oganov, E. Ilyina-KaKUEVA, J. F. MARINI, J. J. LegER, S. C. BODINE-Fowler, AND V. R. EDGERTON. Metabolic and morphologic properties of single muscle fibers in the rat after spaceflight, Cosmos 1887. FASEB J. 4: $64-72,1990$.

29. Overton, J. M., AND C. M. TiPTon. Effect of hindlimb suspension on cardiovascular responses to sympathomimetics and lower body negative pressure. J. Appl. Physiol. 68: 355-362, 1990.

30. Overton, J. M., C. R. Woodman, and C. M. Tipton. Effect of hindlimb suspension on $\mathrm{V}_{\mathrm{O}_{2} \max }$ and regional blood flow responses to exercise. J. Appl. Physiol 66: 653-659, 1989.

31. Popovic, V. Antiorthostatic hypokinesia and circulation in the rat (Abstract). Physiologist 24: S15-S16, 1981.

32. Saltin, B., G. Blomquist, J. H. Mitchell, R. L. Johnson, K. 
WiLdenthal, AND C. B. Chapman. Response to exercise after bed rest and after training. Circulation 38: 1-78, 1968.

33. Smith, J. L., V. R. Edgerton, B. BetTs, and T. C. Collatos. EMG of slow and fast ankle extensors of cat during posture, locomotion, and jumping. J. Neurophysiol. 40: 503-513, 1977.

34. Templeton, G. H., M. Padalino, J. Manton, M. Glasberg, C. J. Silver, P. Silver, G. Denartino, T. Leconey, G. Klug, H. HAGLER, AND J. L. SUTKO. Influence of suspension hypokinesia on rat soleus muscle. J. Appl. Physiol. 56: 278-286, 1984.

35. Templeton, G. H. H. L. Sweeney, B. F. Timson, M. Padalino, AND G. A. DUDENHOEFFER. Changes in fiber composition of soleus muscle during rat hindlimb suspension. J. Appl. Physiol. 65: 11911195,1988

36. WEST, J. B. Spacelab: the coming of age of space physiology research. J. Appl. Physiol. 57: 1625-1631, 1984.

37. Witzman, F. A., D. H. Kim, and R. H. FitTs. Effect of hindlimb immobilization on the fatigability of skeletal muscle. J. Appl. Physiol. 54: 1242-1248, 1983.

38. Yamaguchi, I., T. Torda, F. Hirata, and I. J. Kopin. Adrenoceptor desensitization after immobilization stress or repeated injection of isoproterenol. Am. J. Physiol. 240 (Heart Circ. Physiol. 9): H691-H696, 1981.

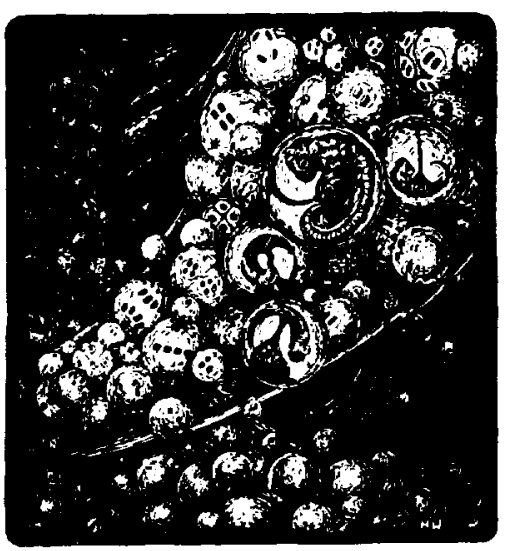

